\title{
Corrigendum
}

\section{Dietary intake of fat and fibre according to reference values relates to higher gut microbiota richness in overweight pregnant women - CORRIGENDUM}

\author{
Henna Röytiö, Kati Mokkala, Tero Vahlberg and Kirsi Laitinen
}

(First published online [13 September 2017])

doi: https://doi.org/10.1017/S0007114517002100

In the original article, the Spearman's correlation coefficients in Table 2 (page 347) are wrongly stated positive. All the coefficient values in this table should have been negative and the table has therefore been amended accordingly. This error does not change the scientific conclusions of the article in any way. We sincerely apologize for this typographic error.

Original:

Table 2. Statistically significant correlations between dietary intakes of nutrients and glycoprotein acetylation ( $n$ 95)

\begin{tabular}{lccc}
\hline & $r^{*}$ & Unadjusted $P$ & Adjusted $P$ 十 \\
\hline Energy & 0.245 & 0.02 & 0.03 \\
Fibre total & 0.316 & 0.002 & 0.01 \\
Non-soluble fibre & 0.353 & $<0.001$ & 0.009 \\
Soluble fibre & 0.323 & 0.001 & 0.01 \\
Protein & 0.301 & 0.003 & 0.01 \\
PUFA & 0.244 & 0.02 & 0.03 \\
$n-3$ LC-PUFA & 0.291 & 0.004 & 0.01 \\
MUFA & 0.230 & 0.02 & 0.04 \\
Vitamin E & 0.279 & 0.006 & 0.02 \\
Pyridoxine & 0.303 & 0.003 & 0.01 \\
Niacin & 0.302 & 0.003 & 0.01 \\
Riboflavin & 0.262 & 0.01 & 0.03 \\
Thiamine & 0.233 & 0.02 & 0.04 \\
Cu & 0.004 & 0.01 \\
Ca & 0.293 & 0.008 & 0.02 \\
F & 0.270 & 0.001 & 0.009 \\
Fe & 0.343 & 0.004 & 0.01 \\
Mg & 0.289 & 0.001 & 0.009 \\
Mn & 0.351 & 0.02 & 0.03 \\
K & 0.246 & 0.003 & 0.01 \\
Se & 0.307 & 0.01 & 0.03 \\
Zn & 0.256 & 0.01 & 0.03 \\
\hline
\end{tabular}

LC-PUFA, long-chain PUFA.

* Spearman's correlation coefficient.

$\dagger$ Benjamini-Hochberg-adjusted $P$ value. 
Correction:

Table 2. Statistically significant correlations between dietary intakes of nutrients and glycoprotein acetylation ( $n$ 95)

\begin{tabular}{|c|c|c|c|}
\hline & $r^{*}$ & Unadjusted $P$ & Adjusted $P+$ \\
\hline Energy & -0.245 & 0.02 & 0.03 \\
\hline Fibre total & -0.316 & 0.002 & 0.01 \\
\hline Non-soluble fibre & -0.353 & $<0.001$ & 0.009 \\
\hline Soluble fibre & -0.323 & 0.001 & 0.01 \\
\hline Protein & -0.301 & 0.003 & 0.01 \\
\hline PUFA & -0.244 & 0.02 & 0.03 \\
\hline$n-3$ LC-PUFA & -0.291 & 0.004 & 0.01 \\
\hline MUFA & -0.230 & 0.02 & 0.04 \\
\hline Vitamin E & -0.279 & 0.006 & 0.02 \\
\hline Pyridoxine & -0.303 & 0.003 & 0.01 \\
\hline Niacin & -0.302 & 0.003 & 0.01 \\
\hline Riboflavin & -0.262 & 0.01 & 0.03 \\
\hline Thiamine & -0.233 & 0.02 & 0.04 \\
\hline $\mathrm{Cu}$ & -0.293 & 0.004 & 0.01 \\
\hline $\mathrm{Ca}$ & -0.270 & 0.008 & 0.02 \\
\hline $\mathrm{F}$ & -0.343 & $<0.001$ & 0.009 \\
\hline $\mathrm{Fe}$ & -0.289 & 0.004 & 0.01 \\
\hline $\mathrm{Mg}$ & -0.351 & $<0.001$ & 0.009 \\
\hline $\mathrm{Mn}$ & -0.246 & 0.02 & 0.03 \\
\hline $\mathrm{K}$ & -0.307 & 0.003 & 0.01 \\
\hline $\mathrm{Se}$ & -0.256 & 0.01 & 0.03 \\
\hline $\mathrm{Zn}$ & -0.256 & 0.01 & 0.03 \\
\hline
\end{tabular}

LC-PUFA, long-chain PUFA

* Spearman's correlation coefficient.

$\dagger$ Benjamini-Hochberg-adjusted $P$ value. 